\title{
Ortsaufgelöste Temperaturmessung mit Hilfe der Kernspintomographie
}

\author{
Hampke M., Busch M.* , Chung Y.**, Wendt M.** , Kraft M., Boenick U. \\ Institut für Mikrotechnik und Medizintechnik, Technische Universität Berlin, Dovestr. 6, 10587 Berlin \\ * Fa. Mediport Counsult, Berlin \\ ** University Hospitals of Cleveland, Ohio, USA
}

\section{EINLEITUNG}

Eine weitverbreitete Methode zur Behandlung nicht operabler Tumore ist die Hyperthermie. Eine Möglichkeit während dieser Behandlung die Temperatur zu kontrollieren, bietet die Kernspintomographie. Ihre Vorteile sind die gute Ortsauflösung, der gute Weichteilkontrast, die beliebige Wahl des Schnittbildverlaufs und ihr geringes Risiko für Nebenwirkungen. Innerhalb dieses Beitrags wird die Entwicklung einer quantitativen ort- und zeitaufgelösten Temperaturmessung im MR an einem gewebenahen Phantom vorgestellt.

\section{MATERIAL UND METHODE}

Es gibt verschiedene Methoden die Temperaturverteilung mit Hilfe der Kernspintomographie zu ermitteln. Die meisten MR Parameter wie die Gleichgewichtsmagnetisierung $\mathrm{M}_{0}$, die Spin-Gitter-Relaxationszeit $\mathrm{T} 1$ und die Spin-Spin-Relaxationszeit T2 zeigen eine Temperaturabhängigkeit. Methoden, die diese Parameter zur Temperaturbestimmung nutzen, sind allerdings ungenau, da es schwierig ist, einen Parameter einzeln zu messen und sie zusätzlich gewebeabhängig sind. Als weitere Verfahren kommen die Messung der chemischen Verschiebung der Wasserstoffprotonen oder des Diffusionskoeffizienten in Frage. Für die Ermittlung des letztgenannten Koeffizienten sind allerdings mehrere Messungen notwendig und zusätzlich muß die gewebe- und temperaturabhängige Aktivierungsenergie bestimmt werden [1].

Die chemische Verschiebung von Wasser hingegen ist proportional zur Temperatur und beträgt $-0,01 \mathrm{ppm} /{ }^{\circ} \mathrm{C}$. Um den durch diesen geringen temperaturabhängigen Koeffizienten hervorgerufenen Effekt zu ermitteln, muß ein zeitlich stabiles und örtlich homogenes äußeres Magnetfeld vorhanden sein. Die Vorteile dieser Methode sind eine Gewebeunabhängigkeit und, im Vergleich zur Diffusionsmessung, die Bekanntheit aller Parameter der Formel. Die Temperaturdifferenz $\Delta T$ wird aus der Phasendifferenz eines Bildes nach einer Temperaturänderung $\phi(T(r))$ und eines Referenzbildes mit bekannter Temperatur $\phi\left(T_{0}(r)\right)$ folgendermaßen bestimmt [1]:

$$
\Delta T=\frac{\Phi(T(r))-\Phi\left(T_{0}(r)\right)}{\alpha \gamma B_{0} T E} .
$$

Hierbei ist $\alpha$ die chemische Verschiebung, $\gamma$ das gyromagnetische Verhältnis, $B_{0}$ die Feldstärke des äußeren Magnetfelds und TE die Echozeit der Sequenz.

Für die Versuche wurde ein gewebenahes Phantom entwickelt, in dem ein Temperaturgradient eingestellt werden kann. MR-kompartible Thermoelemente überwachen die Temperaturgenauigkeit der Kernspintomographiemessung.

Das Phantom befindet sich in einer Plexiglasbox mit drei Kammern, deren Außenwände $10 \mathrm{~mm}$ und Zwischenwände $2 \mathrm{~mm}$ dick sind. Um einen Temperaturgradienten in der mittleren zu erzeugen, werden die äußeren an einen heißen bzw. kalten Wasserkreislauf angeschlossen. In der mittleren Kammer befindet sich Polyacrylamid [2] als Phantommaterial. Das Polyacrylamid ist ein Polymer, das als Basismaterial in Wasser gelöstes Acrylamid, den Starter Ammonium Persulfat und den Katalysator TMEDA enthält. Durch Änderung des Acrylamidanteils können die Eigenschaften des Polymers variiert werden. So ist das Polyacrylamid mit einem höheren Acrylamidanteil fester und die Wärmeleitfähigkeit geringer. Damit diese Meßsubstanz gewebeähnliches Verhalten zeigt, wurden bei 10 Proben mit einem Acrylamidanteil zwischen $6 \%$ und $15 \%$ die Relaxationszeit T2 gemessen. Diese Werte zeigten eine absteigende Reihe von $500 \mathrm{~ms}$ für $6 \%$ iges bis $230 \mathrm{~ms}$ für 15\%iges Polyacrylamid (zum Vergleich: Gewebe besitzt T2-Werte bis $150 \mathrm{~ms}$ ). Die 15\%ige Konzentration wurde ausgewählt, da sie den niedrigsten T2-Wert besitzt und ein noch zähflüssiges Polymer bildet, welches wiedereingebrachte Thermoelemente vollständig umschließen kann. Außerdem befinden sich in der mittleren Kammer fünf $\mathrm{Cu}$-CuNi-Thermoelemente in einem äquidistanten Abstand von 47,5 mm, wobei der erste und der letzte einen Abstand von je $10 \mathrm{~mm}$ zur Zwischenwand hat.

Um eine Temperaturänderung mit der Methode der chemischen Verschiebung zu detektieren, muß die Sequenz sensibel für Phasenänderungen sein, eine lange Echozeit für eine große Phasendifferenz besitzen und eine schnelle Bildgebung ermöglichen. Letztere wird durch eine kurze Repetitionszeit TR (Zeit zwischen zwei Anregungsimpulsen) ermöglicht. Diese Anforderungen erfült die echo-shifted flash sequence, deren Struktur in Abb. 1 dargestellt ist [3]:

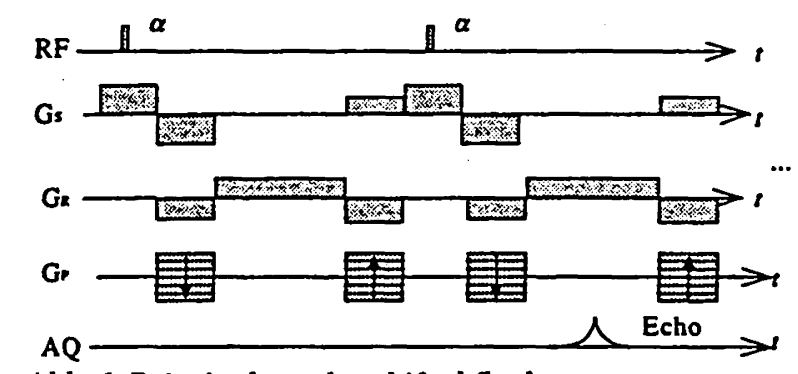

Abb. 1:Prinzip der echo-shifted flash sequence 
Die skizzierte Sequenz wurde in einem Niedrigfeldkernspintomographen mit einer Feldstärke von 0,2-T umgesetzt. Der Hochfrequenzimpuls RF dreht die Spins der gewählten Schichtdicke um den Winkel $\alpha$ in die $x, y$-Ebene. Die Gradienten bewirken eine Dephasierung der Spins. Ein Echo kann aber nur entstehen, wenn diese zum Zeitpunkt der Detektierung des Signals AQ wieder rephasiert wurden. Dies erreicht man, indem der Gradient nach Anregung der Spins mit halber Amplitude umgepolt wird. In Richtung der Schichtselektion wird am Anfang der Repetitionszeit TR der Gradient $\mathrm{G}_{s}$ mit gleicher Amplitude umgekehrt, wodurch die Spins dephasiert sind. Am Ende von TR wiederholt sich der Gradient mit halber Amplitude, so daß die Spins im nächsten TR rephasieren. Somit sind Spins, die von dem HF-Impuls der n-ten Periode angeregt wurden, in der $(n+1)$-ten Periode rephasiert. Die Ermittlung des Entstehungsorts eines Signals erfolgt durch eine Phasen- und Frequenzcodierung. Der Phasencodiergradient $G_{P}$ führt innerhalb jeder TR die Phasencodierung durch. Am Ende wird dieser Gradient umgepolt wiederholt, um eine noch vorhandene Quermagnetisierung zu rephasieren. In der Frequenzcodierrichtung sind die Spins zu erst durch einen umgepolten Gradienten mit halber Amplitude dephasiert, bevor sie in der Mitte des Auslesegradient $G_{R}$ rephasiert werden. Anschließend folgt erneut ein umgepolter Gradient mit halber Amplitude, der die Spins innerhalb der Repetitionszeit rephasiert.

Die Phasendifferenz in der Kernspintomographie wird mit Hilfe von Phasenbildern ermittelt. Eine zweidimensionale Fouriertransformation der aufgenommenen Datensätze ermöglicht die anschließende Berechnung der Amplituden- und Phasenbilder (Abb.2).

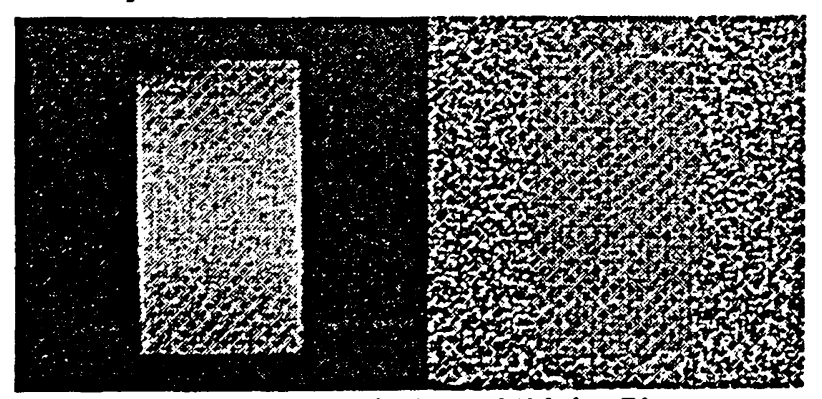

Abb. 2: Amplituden- und Phasenbild des Phantoms

\section{VERSUCHSDURCHFÜHRUNG UND ERGEBNISSE}

$\mathrm{Zu}$ Beginn jedes Versuchs wurde ein Referenzbild bei einer bekannten Temperatur aufgezeichnet. Anschliessend folgte die Erzeugung des Temperaturgradienten im Phantom. Durch Zirkulation von $65^{\circ} \mathrm{C}$ heißem Wasser in einer Außenkammer erhitzte sich die eine Phantomseite. In der anderen Außenkammer zirkulierte ca. $16^{\circ} \mathrm{C}$ kaltes Leitungswasser, wodurch sich die andere Seite des Phantoms abkühlte. Eine Stunde lang erfolgte alle zwei Minuten die Aufzeichnung eines Bildes und die Temperaturmessung mit den Thermoelementen. Mit Hilfe eines IDL-Programms wurde das Referenzphasenbild von einem anderen Meßbild sub. trahiert und nach der obigen Formel die Temperaturdif- ferenz für jeden Pixel berechnet. Der Temperaturverlauf innerhalb des Phantoms kann für jedes Bild ermittelt und sowohl für die Sequenz als auch für die Thermoelemente als Kurve aufgezeichnet werden. Nach einer Stunde ergaben sich folgende Temperaturverläufe in dem Phantom:
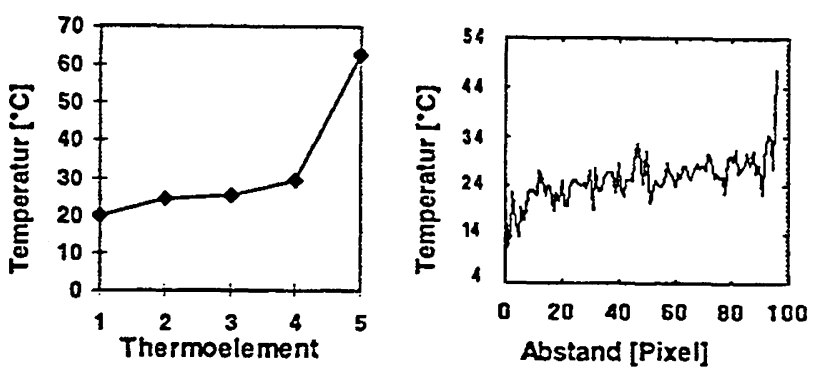

Abb. 3: Temperaturverlauf innerhalb des Phantoms nach einer Stunde Erwärmung und Kühlung ermittelt durch die Thermoelemente und die Sequenz.

Mit einer Anfangstemperatur von $24{ }^{\circ} \mathrm{C}$ erfolgte die Berechnung der mit der Sequenz ermittelten Temperaturkurve in Abb. 3. Es befand sich ca. alle 25 Pixel ein Thermoelement. Vergleicht man die beiden Kurven, stellt man für die Thermoelemente 2, 3 und 4 eine Übereinstimmung der Temperaturwerte fest. So wird mit der Sequenz in diesem Bereich ein Verlauf von $25^{\circ} \mathrm{C}$ bis $29^{\circ} \mathrm{C}$ ermittelt. An den Rändern allerdings erhält man die Anfangstemperatur von $14^{\circ} \mathrm{C}$ und $46^{\circ} \mathrm{C}$ am Ende, wobei dieser Wert nur von einem einzigen Pixel erreicht wird. Beide Werte sind zu niedrig, da die Thermoelemente 1 und 5 eine Temperatur von $20^{\circ} \mathrm{C}$ und $63{ }^{\circ} \mathrm{C}$ erreichten. Legt man eine Ausgleichskurve durch den mit der Sequenz ermittelten Temperaturverlauf, erhält man eine Abweichung der Temperatur von $\pm 5^{\circ} \mathrm{C}$.

\section{DISKUSSION}

Es wurde gezeigt, daß mit der Methode der chemischen Verschiebung eine Temperaturbestimmung mit Hilfe der Kernspintomographie im Phantom möglich ist. An den Rändern des Phantoms konnte jedoch noch keine genaue Messung durchgeführt werden. Eine Ursache dafür kann sein, daß das äußere Magnetfeld des Kernspintomographen nicht homogen ist. Die Phasenverschiebung, die dadurch entsteht, darf nicht in die Temperaturberechnung einfließen. Auch müssen Versuche am lebenden Gewebe zeigen, ob diese Methode übertragbar ist.

\section{LITERATUR}

[1] Ischihara,Y. et al., A prescise and fast temperature mapping using water proton chemical shift; MRM 34 : 814-823 (1995)

[2] Bini, M. et al., The polyacrylamide as a phantom material for electromagnetic hyperthermia studics: IEEE: Vol. BME-31, NO. 3 (1984)

[3] Moonen, C. et al., A fast gradient-recalled MRI technique with increased sensitivity to dynamic susceptibility effects; MRM 26, 184-189 (1992) 